\title{
Implications of prostate-specific antigen screening guidelines on clinical practice at a Canadian regional community hospital
}

\author{
Todd M. Webster, MD, FRCSC'; Erika Lau²; Ken J. Newell, MD PhD FRCPC \\ 'Department of Urology; '2Department of Pathology; Grey Bruce Health Services, Owen Sound, ON, Canada
}

Cite as: Can Urol Assoc J 2017;11 (8):283-6. htrp://dx.doi.org/10.5489/cuaj.4140

\section{Introduction}

Prostate cancer remains the most frequently diagnosed noncutaneous malignancy and the third leading cancer-related cause of death in Canada; however, many men diagnosed with prostate cancer have a good prognosis. ${ }^{1}$ The Canadian Task Force on Preventative Health Care (CTFPHC) produced a guideline in November 2014 on prostate-specific antigen (PSA) screening that was consistent with the 2012 U.S. Preventive Services Task Force (USPSTF) guideline. ${ }^{2,3}$ The Task Force reports that the incidence of prostate cancer increased rapidly subsequent to the widespread adoption of PSA testing, but attributes much of the excess incidence to overdiagnosis, that is, detection of cancers that would not progress or cause symptoms or death. While it would be beneficial to decrease the number of "unnecessary" biopsies by limiting PSA screening - biopsies that carry risks of morbidity and, rarely, mortality but do not detect clinical significant cancers - it would not be desirable to decrease the ability to detect life-threatening cancers at a time when intervention may be curative.

The CTFPHC recommendation is to not screen men of any age for prostate cancer, despite some evidence that screening may improve mortality; ${ }^{4}$ however, much had changed by the time these PSA screening recommendations were published to address overdiagnosis and overtreatment, most importantly the increased tendency to manage men with low-risk prostate cancer with active surveillance (AS). ${ }^{5,6}$ There have been several recent publications, mostly from the U.S., examining the downstream effects of the release of these guidelines on the rate and characteristics of prostate cancer diagnoses. ${ }^{7-13}$ We are confident that most jurisdictions in Canada have been similarly affected and as such we wanted to review our community hospital-based experience with PSA screening and subsequent rate of transrectal ultrasound prostate biopsy (TRUSPB) and radical prostatectomies (RP) to quantify these trends locally.

\section{Our region}

Temporally related to the release of the U.S. and Canadian recommendations, we participated in a South West Local Health Integration Network (SWLHIN) and Cancer Care Ontario (CCO)-led initiative to establish a local Prostate Cancer Diagnostic Assessment Program (PCaDAP), which was launched in 2012. The Grey Bruce Health Services (GBHS) is a 215-bed community hospital in rural Ontario that serves a catchment population of approximately 165 000. The hospital corporation has five sites, one large regional centre and 4 rural sites. Within the catchment area approximately $155000(94 \%)$ of the population has a primary care provider, with 36000 (23\%) being registered at the urban site family health team and the remaining 119 $000(77 \%)$ being registered with the rural site family practice units. PSA testing in Ontario is funded only for patients who have been diagnosed with prostate cancer or in patients with suspicious clinical findings. Patients who present for a PSA screening test in Ontario are required to pay for the testing. PSA screening tests in our region are exclusively ordered by primary care providers, whereas followup PSA tests are predominantly ordered by treating urologists. The GBHS laboratories have been accessioning PSA tests as PSA followup or PSA screening since 2004, as per provincial guidelines. PSA tests are performed on a Beckman-Coulter Unicel DxI immunoanalyzer using the Hydritech PSA methodology. We were able to search this laboratory database for the calendar years 2009, 2011, 2013, and 2015.

Over the same time period, we queried our pathology database for all TRUSPB and RP cases. Biopsies at our site are performed by urology in a standard 12-core fashion and assessed pathologically, as described by Epstein. ${ }^{14}$ The pathologists use a synoptic report that includes criteria established by $\mathrm{CCO}$ and the College of American Pathologists (CAP) guidelines. For each patient having a TRUSPB, data was also retrieved to determine if the biopsy was a first-time biopsy, an AS biopsy in a patient previously diagnosed with prostate cancer, or a repeat biopsy in a patient who had 
had a previous non-malignant result. This chart review was approved by the GBHS ethics review board.

\section{Our regional experience with PSA testing}

The number of men having PSA followup tests has remained relatively constant over time, with approximately 1900 tests being performed per year (Fig. 1). The number of PSA screening tests was relatively constant in 2009 and 2011. There was a $56 \%$ reduction in the number of PSA screening tests from 2011-2015. The most dramatic decrease in the number of PSA screening tests, a 39\% reduction, occurred from 2011-2013. There was also a further 29\% reduction in the number of PSA screening tests from 2013-2015. The reduction in the number of PSA screening tests correlates temporally with the release and promulgation of the USPSTF and CTFPHC guidelines.

This dramatic reduction of PSA testing in our region suggests that the primary care providers and/or patients are well aware of and adhering to the USPSTF and CTFPHC guidelines to not screen men for prostate cancer using PSA. It is possible that these observations are confounded to some degree, as patients registered at the primary care urban site (approximately $20 \%$ of our population) has PSA testing at a private laboratory, and that data was not available for analysis. Furthermore, we have made an assumption of "screening" vs. "followup" testing based on documentation on lab requisitions; however, the dramatic reduction in total number of PSA testing in our region suggests a strong effect of recommendations on the behaviour of primary care physicians around prostate cancer screening.

\section{Our regional experience with TRUSPB}

We were furthermore interested in looking at the downstream effects of this reduction of PSA testing in our region, specifically on the rates of TRUSPB. The mean age of men undergoing biopsy at our institution has shown a small

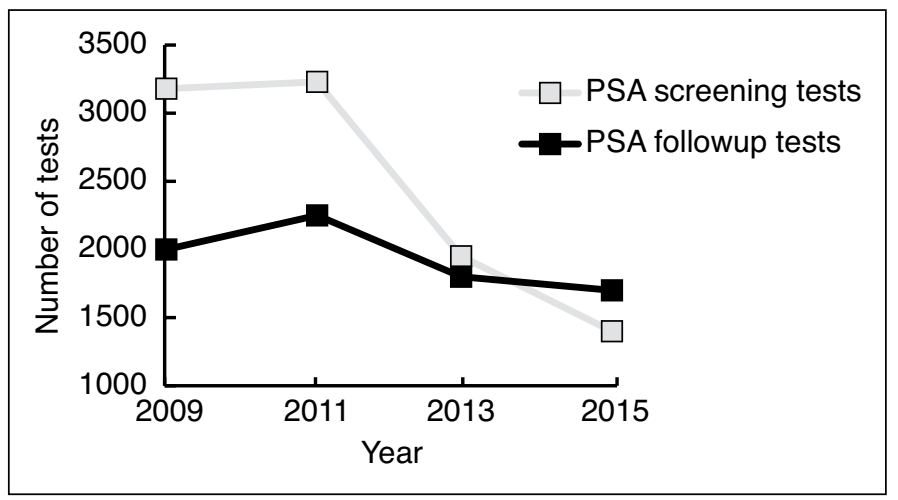

Fig. 1. Numbers of prostate-specific antigen (PSA) followups and PSA screening tests, 2009-2015. decline during the time period from $70.9 \pm 8.1$ years in 2009 to $66.2 \pm 7.0$ years in 2015 . The total number of TRUSPB performed at our institution has decreased $43 \%$ in the sixyear period from 2009-2015 (Fig. 2). Similar to previous studies, we have observed decreased numbers of biopsies following release of the USPSTF guidelines..$^{9,12,13}$ The data from this study shows that the reduction in PSA screening temporally mirrors the reduction in the total number of TRUSPB/number of first-time TRUSPB at our institution.

While the number of TRUSPB performed at our institution has been decreasing over time, the proportion diagnosed with cancer has increased over time from 53\% in 2009 to $70 \%$ in 2015 . Previous data documenting cancer detection rates at TRUSPB in large populations in the range of $30-40 \% 15,16$ would suggest that our high and increasing cancer detection rate may be due to the effects of less PSA screening in our region with higher relative rates of presentation of more advanced disease and/or selectivity of patients and urologists in deciding to go forward with a biopsy.

The rate of Gleason score 6 cancer in positive TRUSPB at our institution has remained fairly stable over time (Table 1 ), although there was some relative decrease from $50 \%$ (48/96) in 2013 to $24 \%$ (23/97) in 2015, with a concomitant increase in the number of Gleason score 7 cancers of $32 \%$ (31/96) in 2013 to $59 \%$ (57/97) in 2015. The relative rate of Gleason score 8,9 , or 10 cancers diagnosed in positive TRUSPB has remained relatively stable over time $(16 \%$ to $18 \%)$. Relative to the total number of biopsies performed per year, we see a slight trend of more significant cancers (33\% in 2009 to $53 \%$ in 2015). Previous studies have raised concern that decreased PSA screening is leading to missing the diagnosis of higher Gleason score cancers at a time when they are curable. ${ }^{9,13}$ Our findings are unable to clearly address these concerns, given the small absolute number of biopsies and cases. As well, recent changes in pathological scoring and review of TRUSPB, combined with the promulgation of the CCO AS guideline and more confirmatory biopsies, has undoubtedly had an effect on these findings at TRUSPB in our institution. ${ }^{17}$

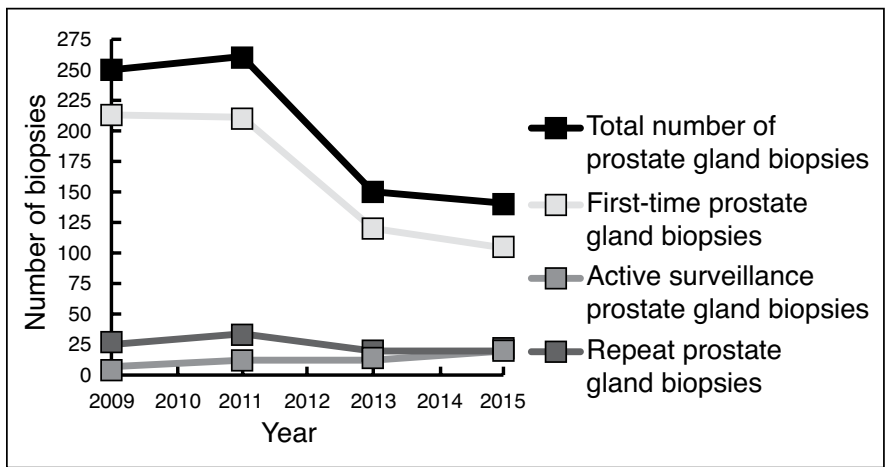

Fig. 2. Numbers of prostate-specific antigen (PSA) followups and PSA screening tests, 2009-2015. 


\begin{tabular}{|c|c|c|c|c|c|}
\hline \multirow[b]{2}{*}{ Year } & \multicolumn{3}{|c|}{$\begin{array}{c}\text { Gleason score in positive } \\
\text { biopsies, } \mathbf{n}(\%)\end{array}$} & \multirow[b]{2}{*}{$\begin{array}{l}\text { Overall total } \\
\text { \# biopsies }\end{array}$} & \multirow[b]{2}{*}{$\begin{array}{c}\text { Total \# (\%) } \\
\text { positive biopsies }\end{array}$} \\
\hline & GS 6 & GS 7 & GS $8,9,10$ & & \\
\hline 2009 & $49(38)$ & $57(44)$ & $24(18)$ & 246 & $130(53)$ \\
\hline 2011 & $54(41)$ & $58(44)$ & $21(16)$ & 258 & $133(52)$ \\
\hline 2013 & $48(54)$ & $31(32)$ & $17(18)$ & 151 & $96(64)$ \\
\hline 2015 & $23(24)$ & $57(59)$ & $17(18)$ & 139 & $97(70)$ \\
\hline
\end{tabular}

\section{Our regional experience with $\mathbf{R P}$}

The mean age of men undergoing RP has been relatively stable over time (overall average 65.2 years). The number of men diagnosed with cancer in TRUSPB at our institution proceeding to RP locally has been relatively stable at approximately $30 \%$; however, the number of RP at our institution has decreased by 31\% from 2013-2015. Data obtained from $\mathrm{CCO}^{18}$ indicates that provincially the number of prostatectomies has fallen by $19 \%$ in the three-year period $2011 / 12$ to $2013 / 14$ (2964 in 2011/12 to 2415 in 2013/14). The number of men having RP for low-grade disease at our institution has fallen steadily from $33 \%$ in 2007 to none in 2015 , consistent with the adoption of AS guidelines. One outcome of this review has been the revision of our DAP data collection tool to be able to capture prospectively the number of referrals, the indication for referral (PSA value, digital rectal exam findings), decision to biopsy, biopsy results, and treatment decision. More fulsome data from our DAP clinic will allow us to better monitor trends and volumes, thereby improving resource planning and allocation. We look forward to sharing this enhanced electronic data collection tool with other DAP clinics and provincial leads with CCO.

\section{Our interpretation}

There have been several other publications that have addressed the impact of PSA testing recommendations. Cohn et al showed a significant decrease in PSA screening by primary care physicians comparing the six-month periods pre- and post-release of the USPSTF guideline. ${ }^{7}$ Jemal et al reported decreased PSA screening and decreased numbers of early-stage cancers being diagnosed in men greater than 50 years old in the year following release of the USPSTF guideline. ${ }^{8}$ Barocas et al identified a $28 \%$ reduction in the incident diagnosis of prostate cancer in the year after the release of the USPSTF guideline. ${ }^{9}$ Drazer et al showed a significant decrease in PSA screening in men 50-59 years old 60-74 years old, and 75 years or older using the National Health Interview Survey data set. ${ }^{10}$ Of concern, Sammon et al, also using the National Health Interview Survey data set from 2005, 2010, and 2013, reported a significant decrease in the proportion of men 50-74 years old undergoing PSA screening, but no significant decrease in PSA screening in men 75 years or older, a cohort at high-risk of overdiagnosis. ${ }^{11}$ Banerji et al reported a $31 \%$ reduction in the absolute number of prostate gland biopsies with a 1.25 increased relative risk of being diagnosed with high-risk prostate cancer in the two and a half years after the USPSTF guideline. ${ }^{12}$

One significant concern of some of these studies was the finding that the majority of the decrease occurred in the detection of intermediate-risk tumours. Bhindi et al performed a time series analysis that showed a decrease in the total number of prostate gland biopsies. ${ }^{13}$ The median number of biopsies decreased from 58 to 35.5 per month, with first-time biopsies decreasing from a median of 42.5 to 24. Fewer low-risk cancers were detected (median number of low-risk prostate cancers detected per month decreased from 8.5 (interquartile range [IQR] 6.5-10.5) to 5.5 (IQR 4.0-7.0) ( $p=0.012)$, while the median number of intermediate-to high-grade prostate cancers per month decreased from 17.5 (IQR 14.5-21.5) to 10.0 (IQR 9.0-12.0) $(\mathrm{p}<0.001)$.

These publications provide evidence that the release of the USPSTF and CTFPHE recommendations has been associated with decreased PSA screening, decreased numbers of prostate gland biopsies, decreased numbers of cancers being diagnosed, and an unclear but potential effect on the risk of missing the diagnosis of high-risk prostate cancer at a time when it is curable.

The results of this review of our DAP highlight the shifting trends in PSA screening, as well as in the assessment and treatment of men for and with prostate cancer. We identified a $56 \%$ decrease in the number of men undergoing PSA screening, a 54\% decrease in the total number of TRUSPB, and a $51 \%$ decrease in the number of first-time TRUSPB from 2009-2015. We have also demonstrated a 31\% decrease in the number of men undergoing RP at our institution during the period 2013-2015. We believe the decline in numbers of men undergoing PSA screening, TRUSPB, and RP could have a profound impact at the local, provincial, and national levels for urological service resource allocation.

Competing interests: The authors report no competing personal or financial interests.

Acknowledgements: We would like to thank Mr. J. Amrhein, M Stat P Stat, for critical review of the manuscript. We would also like to thank Ms. C. Fenton-Stone, Ms. P. Maloney, and Ms. S. Glass for assistance with this project.

This paper has been peer-reviewed. 


\section{References}

1. Canadian Cancer Society Advisory Committee on Cancer Statistics. Canadian Cancer Statistics 2013. Toronto: Canadian Cancer Society; 2013.

2. Canadian Task Force on Preventive Health Care, Bell N, Connor Gorber S, Shane A, et al. Recommendations on screening for prostate cancer with the prostate-specific antigen test. CMAJ 2014;186:1225-34.

3. Moyer VA, U.S. Preventive Services Task Force. Screening for prostate cancer: U.S. Preventive Services Task Force recommendation statement. Ann Intern Med 2012;157:120-34. https://doi.org/10.7326/00034819-157-2-201207170-00459

4. Schröder FH, Hugosson J, Carlsson S, et al. Screening for prostate cancer decreases the risk of developing metastatic disease: Findings from the European Randomized Study of Screening for Prostate Cancer (ERSPC). Eur Urol 2012;62:745-52. https://doi.org/10.1016/i.eururo.2012.05.068

5. Morash C, Tey R, Agbassi C,et al. Evidence-based series 17-9, A quality initiative of the Program in Evidence-Based Care (PEBC), Cancer Care Ontario (CCO). Active Surveillance for the Management of Localized Prostate Cancer. 2014. Available at hitps://www.cancercare.on.ca/common/pages/UserFile. aspx?fileld=325696. Accessed July 10, 2017.

6. Cancer Care Ontario (CCO). Surgical oncology: Performance of needle biopsy of the prostate for men with suspected or established prostate cancer. March 312014

7. Cohn JA, Wang CE, Lakeman JC, et al. Primary care physician PSA screening practices before and after the final U.S. Preventive Services Task Force recommendation. Urologic Oncol 2014;32:e23-41. https://doi.org/10.1016/i.urolonc.2013.04.013

8. Jemal A, Fedewa SA, Ma J, et al. Prostate cancer incidence and PSA testing patterns in relation to USPSTF screening recommendations. JAMA 2015;314:2054-61. https://doi.org/10.1001/jama.2015.14905

9. Barocas DA, Mallin K, Graves AJ, et al. Effect of the USPSTF grade D recommendation against screening for prostate cancer on incident prostate cancer diagnoses in the United States. J Urol 2015;194:1587-93. https://doi.org/10.1016/i.juro.2015.06.075

10. Drazer MW, Huo D, Eggener SE. National prostate cancer screening rates after the 2012 US Preventive Services Task Force recommendation discouraging prostate-specific antigen-based screening. J Clin Oncol 2015;33:2416-23. https://doi.org/10.1200/JC0.2015.61.6532
11. Sammon JD, Abdollah F, Choveiri TK, et al. Prostate-specific antigen screening after 2012 US Preventive Services Task Force recommendation. JAMA 2015;314:2077-9. https://doi.org/10.1001/ jama.2015.7273

12. Banerii IS, Wolff EM, Massman JD, et al. Prostate needle biopsy outcomes in the era of the U.S. Preventive Services Task Force recommendation against prostate specific antigen screening. J Urol 2016;195:66-73. https://doi.org/10.1016/i.juro.2015.07.099

13. Bhindi B, Mamdani M, Kulkarni GS, et al. Impact of the U.S. Preventive Services Task Force recommendation against prostate-specific antigen screening on prostate biopsy and cancer detection rates. J Urol 2015;193:1519-24. https://doi.org/10.1016/i.juro.2014.11.096

14. Epstein Jl. Pathological assessment of the surgical specimen. Urol Clin North Am 2001;28:567-94. https://doi.org/10.1016/S0094-0143(05)70164-6

15. Siegel R, Ma J, Zou Z, et al. Cancer statistics, 2014. CA Cancer J Clin 2014;64:9-29. https://doi.org/10.3322/caac.21208

16. Catalona WJ, Richie JP, deKernion JB, et al. Comparison of prostate-specific antigen concentration vs. prostate-specific antigen density in the early detection of prostate cancer: Receiver operating characteristic curves. J Urol 1994;152:2031-6. https://doi.org/10.1016/S0022-5347(17)32299-1

17. Amin MB, Lin DW, Gore JL, et al. The critical role of the pathologist in determining eligibility for active surveillance as a management option in patients with prostate cancer: Consensus statement with recommendations supported by the College of American Pathologists, International Society of Urological Pathology, Association of Directors of Anatomic and Surgical Pathology, the New Zealand Society of Pathologists, and the Prostate Cancer Foundation. Arch Pathol Lab Med 2014;138:1387-405. https://doi.org/10.5858/arpa.2014-0219-SA

18. Morash C, McKnight L. Ontario Prostatectomy numbers, DAD Database, CCO Data, 2014.

Correspondence: Dr. Todd M. Webster, Department of Urology, Grey Bruce Health Services, Owen Sound, ON, Canada; twebster@gbhs.on.ca 